\title{
Reviewers of ASHS Manuscripts 2005
}

ASHS is pleased to publish the following list of members and other individuals who reviewed manuscripts for the Journal. The Society appreciates the time and unselfish effort rendered by reviewers and acknowledges their contribution in making ASHS publications scientifically reliable and useful.

\begin{tabular}{|c|c|c|}
\hline Abbott, Albert G. & Brown, Jack & Dickinson, Timothy \\
\hline Adelberg, Jeff & Brown, Kathleen & Dixon, Jonathan \\
\hline Adkins, Jeffrey & Brown, Rebecca & Dole, John \\
\hline Albano, Joseph & Brown, Robert & Domoto, Paul \\
\hline Albertsen, Marc & Brown, Susan K. & Dong, Shufu \\
\hline Albrigo, Leo G. & Bryla, David & Dorais, Martine \\
\hline Aldwinkle, Herbert S. & Buchner, Richard & Dosman, Michael S. \\
\hline Alice, Lawrence & Burns, Jackie K. & Douches, David S. \\
\hline Alyokhin, Andrei & Byrne, David H. & Drake, Steve \\
\hline Amita Pal, Amita & Callahan, Ann & Dukes, Michael \\
\hline Anagnostakis, Sandra & Cameron, Art & Durieux, Robert \\
\hline Anderson, Jeff & Carde, Jean-Pierre & Duval, John \\
\hline Anella, Louis & Carena, Marcelo & Earle, Elizabeth \\
\hline Aradhya, Mallikarijuna K. & Carle, Bruce & Eason, Jocelyn \\
\hline Archbold, Douglas & Carson, Martin L. & Eaton, Greg \\
\hline Arnold, Claire & Cawly, John D. & Echt, Craig \\
\hline Arnold, Michael A. & Chao, Chih-Cheng T. & Elliott, George \\
\hline Arora, Rajeev & Chaparro, Jose & Ernest, Emmalea Garver \\
\hline Arpaia, Mary Lu & Charlesworth, Deborah & Etxeberria, Ed \\
\hline Artlip, Timothy S. & Charron, Craig S. & Evans, Richard \\
\hline Auge, R.M. & Chen, Jianjun & Eveleens-Clark, Barbara \\
\hline Augustine, Jimmy & Chen, Jianjun & Fahey, Jed \\
\hline Ayars, James & Chen, Jiquan & Fain, Glenn \\
\hline Bai, Jinhe & Cheng, Lailiang & Fallahi, Esmaeil \\
\hline Bakry, Frederic & Chetelat, Roger & Fare, Donna \\
\hline Baldoni, Luciana & Cisnero-Zevallos, Luis & Farnham, Mark \\
\hline Baldwin, Elizabeth A. & Clark, David & Faust, Jim \\
\hline Baligar, Virupax & Clark, John R. & Fazio, Gennaro \\
\hline Ballington, James & Clarke, Jihong & Ferguson, James J. \\
\hline Bar-Tal, Asher & Clement, Didier & Ferguson, Louise \\
\hline Barry, Graham & Clements, Michael J. & Fernandez, George \\
\hline Bassett, Carole & Cohen, Yuval & Fernández-Muñoz, Rafael \\
\hline Bassett, Mark J. & Coleman, David & Fery, Richard L. \\
\hline Bassil, Nahla V. & Coleman, Gary & Fidelibus, Matthew W. \\
\hline Bauerle, William L. & Colova, Violetka M. & Foolad, Majid \\
\hline Beaudry, Randolph & Compton, Michael & Fowler, James L. \\
\hline Beaver, James & Contreras, Samuel & Fox, Kevin \\
\hline Bedgood, Dan & Costa, Heather S. & Francis, David M. \\
\hline Behboudian, Mohammad & Craddock, J. H. & Frantz, Jonathan M. \\
\hline Bell, Richard & Cregg, Bert & Funnell, Keith A. \\
\hline Benkeblia, Noureddine & Crowe, Fred & Galiba, Gábor \\
\hline Bentz, Jo-Ann & Cuevas, Julien & Gardea, Jorge \\
\hline Bervillé, André & Curry, Eric A. & Gardiner, Michele \\
\hline Bhardway, Harbans & Dag, Arnon & Gardner, David S. \\
\hline Bi, Guihong & Dale, Adam & Gardner, Randolph \\
\hline Binder, Brad & Dandekar, Abhaya M. & Gatehouse, John \\
\hline Bishop, Andrea & Dane, Fenny & Gaudillere, Jean Pierre \\
\hline Bishop, Bert & Darnell, Rebecca & Geigenberger, Peter \\
\hline Black, Brent L. & Daugovish, Oleg & Gemma, Hiroshi \\
\hline Bliss, Fred A. & Davenport, Joan & Geneve, Robert \\
\hline Blom, Theo & Davenport, Thomas & Georgi, Laura \\
\hline Bohn, Martin & Davies, Frederick S. & Giordani, Edgardo \\
\hline Bolyard, Mark & Davis, Angela & Girona, Joan \\
\hline Bongi, Guido & Davis, Tom M. & Giuliano, Giovanni \\
\hline Bonos, Stacey & De la Rosa, Raul & Glozer, Kitren \\
\hline Bordelon, Bruce & De Pascale, Stefania & Goffreda, Joseph C. \\
\hline Bosland, Paul W. & De Riek, Jan & Goldman, Irwin L. \\
\hline Both, A.J. & Debener, Thomas & Goldschmidt, Eliezer \\
\hline Botta, Roberto & Defilippi, Bruno & Goldway, Martin \\
\hline Bourne, Timothy & DeJong, Ted M. & Goss, Ryan \\
\hline Bradford, Kent & Deng, Zhanao & Gottlieb, Eric \\
\hline Brand, Mark & DePascale, Stefanie & Graetz, Donald A. \\
\hline Brevis, Patricio A. & Deyholos, Michael & Graham, Elaine \\
\hline Briggs, Winslow & Deyton, Dennis & Graham, Julie \\
\hline Brooking, Ian & Dickinson, Matthew & Granger, Andrew \\
\hline
\end{tabular}

Gray, Dennis J.

Griesbach, Robert J.

Griffis, John

Griffiths, Phillip D.

Groff, David

Grosser, Jude W.

Grube, Rebecca

Grumet, Rebecca

Grusak, Michael

Gucci, Riccardo

Guo, W.W.

Gusmini, Gabriele

Gusta, Larry

Halberman, Dennis

Hale, Anna L.

Hancock, James F.

Hanson, Blaine

$\mathrm{Hao}, \mathrm{Yu}$

Hashimoto, Atsushi

Havey, Michael J.

Haynes, Kathleen

Heckman, Joseph

Hellier, Barbara

Hemphill, Delbert

Henny, Richard J.

Hernandez-Sancho, F.

Heuvelink, Ep

Hicklenton, Peter

Hill, Hank

Hodges, D.M.

Hokanson, Stan C.

Hongwen, Huang

Horgan, Graham W.

Hull, Richard J.

Hummel, Rita

Hummer, Kim

Iezzoni, Amy

Iglesias, Domingo

Irish, Bryan

Irving, Donald E.

Jack, Thomas

Jamieson, Peter

Jiang, Yiwei

Jifon, John L.

Johnson, Duane L.

Johnson, Scott

Jones, Michelle L.

Jones, Richard

Kafkafi, Uzi

Kallsen, Craig E.

Kalt, Wilhelmina

Kamenetsky, Rina

Kane, Michael E.

Kang, Manjit

Karlson, Dale

Karlsson, Meriam

Kelly, James

Khan, Iqrar

Khan, Muhammad M.

Kim, SunGil

King, Stephen R.

Kirkham, Mary Beth

Klein, Isaac

Kleinhenz, Matthew D.

Klotz, Karen 
Knowles, N. Richard

Kobayashi, Kent

Korban, Schuyler

Kosola, Kevin

Kramer, Matthew

Krueger, Robert R.

Kubisiak, Thomas L.

Kuhar, Tom

Kwong, Francis

Lang, Ping

Larrigaudière, Christian

Lavee, Shimon

Lavi, Uri

Layne, Desmond R.

Leskovar, Daniel I.

Lester, Gene E.

Levi, Amnon

Lichter, Amnon

Lightfoot, David

Lineberger, R.D.

Lingle, Sarah

Liu, Weixin

Lo Bianco, Ricardo

Lobell, David

Loescher, Wayne $\mathrm{H}$.

Lombardini, Leonardo

Louzada, Eliezer

Lownds, Norman

Loy, Brent

Luby, Jim

Luo, Yaguang

Mable, Barbara

Mackay, Wayne A.

Mackey, Bruce

Mahuku, George

Maki, Sonja

Marin, Juan A

Marini, Richard

Maroon-Lango, Clarissa

Marsal, Jordi

Marshall, Donna

Martin, Jack

Martinez, Pedro Florian

Martinez-Gomez, Pedro

Mathers, Hannah

Matsumoto, Tracie

Mattheis, James

McClean, Phil E.

McConnell, James

McCormick, Sheila

McCown, Brent

McCreight, James D.

McDonald, Louis M.

McGovern, Robert

McMahon, Margaret J.

McNeilage, Mark

Mehlenbacher, Shawn A.

Merhaut, Donald

Mes, Peter

Mickelbart, Michael V.

Mielke, Eugene

Mikkelsen, Robert L.

Miller, Greg

Miller, Steve

Miller, William B.

Millner, Pat

Mitchell, Cary A.

Mok, David

Monteiro, Jose

Moran, Renae

Mou, Beiquan

Muleo, Rosario
Muller, Renate

Munshaw, Gregg

Muthukrishnan, Subbaratnam

Myers, James

Myrold, David D.

Mínguez-Mosquera, Maria-Isabel

Nadgauda, Rajani

Nagata, Russel

Nagegowda, Dinesh

Neilsen, Denise

Neri, Davide

NeSmith, Scott

Ngouajio, Mathieu

Niemiera, Alex

Nienhuis, James

Niu, Genhua

Nolte, Phillip

Nonogaki, Hiro

Norcini, Jeffrey

Norelli, Jay L.

Novello, Vittorino

O'Hare, Tim

Obenland, David

Oelke, Lisa

Oki, Lorence

Orbovic, Vladimir

Ortiz, Rodomiro

Orzolek, Michael

Osborne, Dennis

Ouedraogo, Jeremy

Ourry, Alain

Ozga, Jocelyn A.

Paek, Kee-Yoeup

Pallardy, Stephen

Paparozzi, Ellen T.

Parfitt, Dan

Pasian, Claudio

Patterson, Sara

Paull, Robert

Pavel, Edelgard

Pavlis, Gary

Pemberton, Brent

Pennycooke, Joyce C.

Perica, Slavko

Perkins-Veazie, Penelope

Petracek, Peter

Petrie, Paul

Picazo, Andres J.

Pill, Wallace G.

Pitchay, Dharmalingam S.

Plaisted, Doug

Polashock, James J.

Polito, Vito

Pooler, Margaret

Porch, Tim

Potter, Dan

Potters, Geert

Price, H. James

Prince, James J.

Prior, Ronald

Pritchard, Hugh W.

Pritts, Marvin

Proctor, John T.

Quebedeaux, Bruno

Quiros, Carlos F.

Radovich, Theodore J.

Rajapakse, Nihal

Ramesh, Sunita

Ramming, David

Rapoport, Hava

Raveh, Eran

Ray, Dennis T.
Rayburn, A. Lane

Reed, Sandra

Reese, John

Reid, Michael

Renault, Sylvie

Reustle, Goetz M.

Rinehart, Timothy A.

Robacker, Carol

Roh, Mark

Romero-Severson, Jeanne

Rose, Jocelyn

Rosen, Carl

Roux, Nicholas

Rowland, Lisa J.

Ruiz, Juan M.

Runkle, Erik S.

Russo, Vincent

Ryder, Edward J.

Samac, Deborah

Sanchez, Ana

Sandrock, David R.

Saniewski, Marian

Sansavini, Silviero

Sanz, Carlos

Sanzol, Javier

Satovic, Zlatko

Scagel, Carolyn F.

Schaffer, Bruce

Schnell, Raymond J.

Schotzko, R. Thomas

Schreiner, Paul

Schroeder, Kenneth R.

Schuch, Ursula

Scoggins, Holly

Scott, Tom

Sebastiani, Luca

Sellmer, James

Sharma, Jyotsna

Shaw, Douglas V.

Sheldon, Anna

Shellie, Krista

Shewfelt, Robert

Shirazi, Abbas

Silber, Avner

Silva, Erin M.

Singh, Zora

Siomos, Anastasios

Smilanick, Joseph

Smith, Brandon

Smith, Malcolm

Snapp, Sieglinde

Socias i Company, Rafael

Soleri, Daniela

Song, Jun

Sosinski, Bryon

Sparks, Darrell

Spiers, James

St. Hilaire, Rolston

Staub, Jack E.

Stern, Raffi

Stevens, Mikel

Stewart, Colin

Still, David

Stommel, John R.

Stover, Ed

Struve, Daniel

Stutte, Gary

Sugiyama, Nobuo

Suslow, Trevor

Syvertsen, Jim

Tabuchi, Toshihito

Takeda, Fumiomi
Talbert, Luther

Talon, Manuel

Tamura, Fumio

Tanino, Karen

Tao, Ryutaro

Tattini, Massimiliano

Taylor, Alan

Taylor, Gregory

Taylor, N.J.

Teng, Yuanwen

Terry, Leon

Testolin, Raffaele

Thompson, Thomas

Tognetti, Roberto

Toivonen, Peter

Trappey, Alfred

Trrkan, Osmail

Uemura, Matsuo

Uma, Subbaraya

Van Es, Harold

Van Iersel, Marc

Vanden Heuvel, Justine

Vandenberg, Bert

Vannocker, Steven R.

Vasilakakis, Miltiadis

Velasco, Leonardo

Villordon, Arthur

Vossen, Paul

Waines, Giles

Walcroft, Adrian

Walker, Stephanie

Wall, Marisa M.

Walter, Monika

Wample, Robert

Wang, Ruojing

Wang, Yin-Tung

Wang, Zengyu

Warmund, Michele

Warner, Ryan M.

Watkins, Chris B.

Weber, Courtney A.

Welsh, Molly M.

Werner, Dennis J.

Wessel-Beaver, Linda

Wetzstein, Hazel Y.

Whitaker, Bruce

White, Allan

White, Donald G.

White, Jeff

Whiting, Matthew

Wien, H. Chris

Wiersma, Paul

Williams, Kimberly A.

Williams, Larry

Wisniewski, Michael E.

Wright, Robert D.

Wunsch, Ana

Xiao, Chang-Lin

$\mathrm{Xu}$, Wenwei

Yamane, Hisayo

Yang, Wei Qiang

Yuan, Rongcai

Zewdie, Yayeh

Zhang, Donglin Zhang

Zhang, John

Zhao, Yanyun

Zinati, Gladis

Zitter, Tom

Zobel, Richard W. 